\title{
FADIGA NA FORMA REMITENTE RECORRENTE DA ESCLEROSE MÚLTIPLA
}

\author{
MARIA FERNANDA MENDES**, CHARLES PETER TILBERY*, SILVIA BALSIMELLI****, \\ EDUARDO FELIPE**, MARCOS AURELIO MOREIRA ***, ANA MARIA BARÃO-CRUZ****
}

\begin{abstract}
RESUMO - Foram avaliados 95 pacientes com forma remitente-recorrente da esclerose múltipla quanto à presença de fadiga. A Escala de Severidade de Fadiga foi aplicada em todos os pacientes. Em 64 pacientes $(67,4 \%)$ a fadiga foi encontrada. Não observamos diferenças clínicas quanto ao gênero, idade, grau de incapacidade funcional e depressão, nos pacientes com e sem fadiga. Foi encontrada correlação entre ansiedade e tempo de doença com a presença de fadiga. Ao analisarmos estas variáveis quanto à intensidade da fadiga, observamos haver associação entre fadiga grave e maior incapacidade funcional.
\end{abstract}

PALAVRAS-CHAVE: esclerose múltipla, fadiga, EDSS, depressão, ansiedade.

\section{Fatigue in multiple sclerosis relapsing-remitting form}

ABSTRACT - In 95 patients with the remitting-relapsing form of multiple sclerosis we investigated fatigue. All of them were evaluated with the Fatigue Severity Scale and we found it in 64 patients (67.4\%). Gender, age, depression and fuctional incapacity was not predictive of fatigue occurrence, while anxiety and time of disease seems to be correlated with it. When we analysed the fatigue severity, a correlation between the EDSS and the increasing fatigue severity was found.

KEY WORDS: multiple sclerosis, fatigue, EDSS, depression, anxiety.

A esclerose múltipla (EM) é afecção neurológica desmielinizante, que acomete preferencialmente adultos jovens, evoluindo de forma crônica e imprevisível. Apresenta sinais e sintomas muito variáveis, e tem a fadiga como uma de suas principais características. A definição de fadiga pode ser feita de várias maneiras, permitindo interpretações diferentes. Para os fisiologistas, é considerada como uma dificuldade em manter a contração muscular, podendo ser objetivamente analisada e quantificada. Esta definição considera apenas a fadiga muscular, tornando-se muito limitada na prática clínica. Para os pacientes com EM, a fadiga é um sintoma subjetivo, definido como sensação de cansaço físico ou mental profundo, perda de energia ou mesmo sensação de exaustão, com características diferentes daquelas observadas na depressão ou fraqueza muscular ${ }^{1}$. Nos pacientes com doenças neurológicas, a fadiga é diferente daquela relatada pelos demais, levando a maior comprometimento da qualidade de vida. Na EM é sintoma frequente e incapacitante que acomete de 75 a $95 \%$ dos portadores, não sendo correlacionada com idade, sexo, depressão ou grau de acometimento neurológico ${ }^{2-4}$. Sua mensuração usualmente tem sido relatada através de escalas de auto-avaliação, por tratar-se de sintoma subjetivo ${ }^{5}$. Mendes, Tilbery e Felipe ${ }^{5}$ realizaram estudo preliminar em pacientes com EM, encontrando fadiga em $70 \%$ dos pacientes, porém sem estudar as suas principais correlações clínicas. Não encontramos no nosso meio estudos sobre este sintoma.

O objetivo deste estudo é analisar na nossa população as características da fadiga nos pacientes com EM forma remitente- recorrente (RR), e os principais fatores associados.

Centro de Atendimento e Tratamento de Esclerose Múltipla (CATEM) da Clínica Neurologica do Departamento de Medicina da Faculdade de Ciências Médicas da Santa Casa de São Paulo: *Professor Adjunto; ** Professor Instrutor; ***Médico neurologista; ****Psicóloga. Aceite: 28-fevereiro-2000.

Dra. Maria Fernanda Mendes - Rua Dr. Martinico Prado, 26/122 - 01224-010 São Paulo SP - Brasil. 


\section{MÉTODO}

Foram estudados 95 pacientes com EM forma RR, definidas de acordo com os critérios de Poser e cols. ${ }^{6}$, em acompanhamento no CATEM. No momento do estudo, nenhum dos pacientes encontrava-se em surto da doença e não fazia uso de qualquer medicação depressora do sistema nervoso central.

Em todos os pacientes, independentemente da queixa clínica, a fadiga foi estudada através de escalas de auto-avaliação, com a aplicação da Escala de Severidade de Fadiga (ESF) ${ }^{7}$. O escore superior a 27 é considerado indicativo da presença de fadiga ${ }^{7}$.Quando identificada a fadiga pela ESF, foi aplicada a Escala de Fadiga de Chalder modificada $(\mathrm{EFCm})^{1,8}$, e analisado o escore total e seus sub-itens ${ }^{9}$. No estudo da intensidade da fadiga utilizamos a ESF. Os escores foram definidos arbitrariamente por não haver consenso de literatura a respeito. Consideramos os escores de 28 a 39 como fadiga leve, de 40 a 51 fadiga moderada e de 52 a 63 fadiga grave. As variáveis de gênero, idade, duração da doença, grau de incapacidade física, ansiedade e depressão foram analisadas. O grau de incapacidade funcional foi avaliado pela aplicação do $\operatorname{EDSS}^{10} \mathrm{e}$ a correlação com depressão e ansiedade através da Escala de Ansiedade e Depressão (HAD) ${ }^{11}$.

Os dados foram analisados quanto às possíveis diferenças entre os grupos com e sem fadiga e quanto a interferência destas variáveis na intensidade da fadiga.

\section{RESULTADOS}

Dos 95 pacientes estudados, 79 eram do gênero feminino e 16 do masculino, com média de idade de $34,7( \pm 8,9)$ anos, com faixa etária entre 17 e 57 anos. O EDSS médio foi 2,2 $( \pm 1,5)$. Destes, $21(22,1 \%)$ pacientes tinham EDSS maior ou igual a 3,5 e $74(77,9 \%)$ EDSS menor que 3,5. A depressão foi detectada em 14 (14,7\%) e a ansiedade em 29 (30,5\%) pacientes.

A fadiga foi observada em 64 pacientes $(67,4 \%)$, comprovada pela aplicação da ESF, com escore superior a 27 . Destes pacientes, $53(82,8 \%)$ eram do gênero feminino e $11(17,2 \%)$ do masculino, com média de idade de $36,3( \pm 7,5)$ anos. A ansiedade foi observada em $21(32,8 \%)$ pacientes e a depressão em $10(15,6 \%)$. O escore médio foi $6,2( \pm 3,6)$ e $4,2( \pm 3,4)$ para ansiedade e depressão respectivamente. O EDSS médio encontrado foi $2,3( \pm 1,5)$. Destes, $50(78,1 \%)$ tinham escore inferior a 3,5 e $14(21,9 \%)$ escore maior ou igual a 3,5 .

No grupo sem fadiga encontramos $31(32,6 \%)$ pacientes. Características clínicas semelhantes foram observadas nos pacientes com e sem fadiga, exceto quanto à duração da doença, conforme demonstramos na Tabela 1.

Tabela 1. Características clínicas dos pacientes com e sem fadiga.

\begin{tabular}{|c|c|c|c|c|}
\hline Características & & com fadiga & sem fadiga & total \\
\hline & ESF & $\begin{array}{c}n=64(67,4 \%) \\
46,3( \pm 9,4)\end{array}$ & $\begin{array}{c}\mathrm{n}=31(32,6 \%) \\
17,1( \pm 6,9)\end{array}$ & $\begin{array}{c}\mathrm{n}=95(100 \%) \\
36,8( \pm 16,2)\end{array}$ \\
\hline & fem & $53(82,8 \%)$ & $26(83,9 \%)$ & $79(83,2 \%)$ \\
\hline Gênero & $\begin{array}{c}\text { masc } \\
\text { fem/masc }\end{array}$ & $\begin{array}{c}11(17,2 \%) \\
4,8: 1\end{array}$ & $\begin{array}{c}05(16,1 \%) \\
5,2: 1\end{array}$ & $\begin{array}{c}16(16,8 \%) \\
4,9: 1\end{array}$ \\
\hline Idade & $\begin{array}{c}\bar{x} \\
\text { faixa }\end{array}$ & $\begin{array}{c}36,3( \pm 7,5) \\
21|-| 49\end{array}$ & $\begin{array}{c}31,3( \pm 11,3) \\
17|-| 57\end{array}$ & $\begin{array}{c}34,7( \pm 8,9) \\
17|-| 57\end{array}$ \\
\hline Duração da doença (meses) & & $75,3( \pm 50,2)$ & $37,7( \pm 37,0)$ & $63,7( \pm 49,7)$ \\
\hline HAD & $\begin{array}{l}\text { ANS x } \\
\text { ANS x } \\
\text { DEP x } \\
\text { DEP x }\end{array}$ & $\begin{array}{c}6,2( \pm 3,6) \\
21(32,8 \%) \\
4,2( \pm 3,4) \\
10(15,6 \%)\end{array}$ & $\begin{array}{c}5,9( \pm 3,6) \\
09(25,8 \%) \\
3,3( \pm 3,3) \\
04(12,9 \%)\end{array}$ & $\begin{array}{c}6,1( \pm 3,6) \\
29(30,5 \%) \\
3,9( \pm 3,4) \\
14(14,7 \%)\end{array}$ \\
\hline EDSS & $\begin{array}{c}\bar{x} \\
n \geq 3,5 \\
n<3,5\end{array}$ & $\begin{array}{l}2,3( \pm 1,5) \\
14(21,9 \%) \\
50(78,1 \%)\end{array}$ & $\begin{array}{l}2,1( \pm 1,7) \\
07(22,6 \%) \\
24(77,4 \%)\end{array}$ & $\begin{array}{l}2,2( \pm 1,5) \\
21(22,1 \%) \\
14(14,7 \%)\end{array}$ \\
\hline
\end{tabular}

ESF, escala de Severidade da fadiga; fem, feminino; masc, masculino; HAD, escala de ansiedade e depressão; ANS, ansiedade; DEP, depressão. 
Tabela 2. Características clínicas quanto a intensidade da fadiga

\begin{tabular}{|c|c|c|c|c|c|}
\hline & & & Fadiga & & \\
\hline \multirow[t]{3}{*}{ Características } & & leve & moderada & grave & total \\
\hline & & $28|-| 39$ & $40|-| 51$ & $52|-| 63$ & \\
\hline & $\mathrm{n}=$ & $19(29,9 \%)$ & $21(32,9 \%)$ & $24(37,5 \%)$ & $64(100 \%)$ \\
\hline \multirow[t]{3}{*}{ Gênero } & fem & $17(89,5 \%)$ & $17(80,9 \%)$ & $19(79,2 \%)$ & $53(82,8 \%)$ \\
\hline & masc & $02(10,5 \%)$ & $04(19,1 \%)$ & $05(20,8 \%)$ & $11(17,2 \%)$ \\
\hline & $\mathrm{fem} / \mathrm{masc}$ & $8,5: 1$ & $4,25: 1$ & $3,8: 1$ & $4,8: 1$ \\
\hline \multirow[t]{2}{*}{ Idade } & $\bar{x}$ & $32,2( \pm 7,5)$ & $36,8( \pm 6,6)$ & $38,0( \pm 6,4)$ & $36,3( \pm 7,0)$ \\
\hline & faixa & $21|-| 47$ & $27|-| 49$ & $24|-| 48$ & $21|-| 49$ \\
\hline \multirow{3}{*}{$\begin{array}{c}\text { Duração da } \\
\text { doença (meses) }\end{array}$} & & $71,2( \pm 47,3)$ & $69,5( \pm 49,0)$ & $84,0( \pm 52,1)$ & $75,3( \pm 50,2)$ \\
\hline & ANS $x$ & $7,2( \pm 3,1)$ & $5,5( \pm 3,8)$ & $6,1( \pm 3,5)$ & $6,2( \pm 3,6)$ \\
\hline & ANS n & $08(42,1 \%)$ & $06(28,6 \%)$ & $07(29,2 \%)$ & $21(32,8 \%)$ \\
\hline \multirow{3}{*}{ HAD } & DEP $x$ & $3,9( \pm 2,8)$ & $4,0( \pm 3,2)$ & $4,7( \pm 3,8)$ & $4,2( \pm 3,4)$ \\
\hline & DEP n & $02(10,5 \%)$ & $04(19,1 \%)$ & $04(16,7 \%)$ & $10(15,6 \%)$ \\
\hline & $\bar{x}$ & $1,8( \pm 1,3)$ & $1,8( \pm 1,4)$ & $3,0( \pm 1,4)$ & $2,3( \pm 1,5)$ \\
\hline \multirow[t]{2}{*}{ EDSS } & $\mathrm{n} \geq 3,5$ & $01(5,3 \%)$ & $04(19 \%)$ & $09(37,5 \%)$ & $14(21,9 \%)$ \\
\hline & $\mathrm{n}<3,5$ & $18(94,7 \%)$ & $17(81 \%)$ & $15(62,5 \%)$ & $50(78,1 \%)$ \\
\hline
\end{tabular}

Legenda: ver Tabela 1.

Ao analisarmos as características clínicas da fadiga quanto à sua intensidade, observamos que 19 pacientes $(29,9 \%)$ apresentavam fadiga leve, 21 (32,9\%) fadiga moderada e $24(37,5 \%)$ fadiga grave. Diferenças foram observadas entre os três grupos, quanto à faixa etária, EDSS e presença ansiedade, conforme demonstramos na Tabela 2.

A EFCm foi aplicada naqueles pacientes com fadiga pela ESF e a fadiga foi observada em apenas 30 pacientes $(46,9 \%)$. Ao analisarmos os sub-itens isoladamente, notamos que $36(56,3 \%)$ apresentavam fadiga física e $21(32,8 \%)$ fadiga mental; nos demais não foi detectada a fadiga por esta escala. O grau de sensibilidade da escala aumenta com a gravidade da fadiga, conforme observamos na Tabela 3.

Tabela 3. Escala de fadiga de Chalder modificada e intensidade da fadiga.

Fadiga

\begin{tabular}{|c|c|c|c|c|c|}
\hline \multirow[t]{4}{*}{ Escala } & & leve & moderada & grave & total \\
\hline & ESF n & $19(29,9 \%)$ & $21(32,8 \%)$ & $24(37,5 \%)$ & $64(100 \%)$ \\
\hline & $\overline{\mathrm{x}}$ & $34,7( \pm 3,9)$ & $45,8( \pm 3,5)$ & $56,0( \pm 3,3)$ & $46,3( \pm 9,4)$ \\
\hline & FIS & $6,3( \pm 2,3)$ & $8,5( \pm 3,1)$ & $10,2( \pm 2,9)$ & $8,5( \pm 3,2)$ \\
\hline Chalder & MENT & $3,5( \pm 2,0)$ & $3,6( \pm 2,5)$ & $4,4( \pm 2,2)$ & $3,9( \pm 2,3)$ \\
\hline \multirow[t]{2}{*}{$\mathrm{x}$} & TOTAL & $9,8( \pm 3,5)$ & $12,1( \pm 4,5)$ & $14,5( \pm 4,4)$ & $12,3( \pm 4,7)$ \\
\hline & FIS & $07(36,8 \%)$ & $11(52,4 \%)$ & $18(75,0 \%)$ & $36(56,3 \%)$ \\
\hline Chalder & MENT & $05(26,2 \%)$ & $06(28,6 \%)$ & $10(41,7 \%)$ & $21(32,8 \%)$ \\
\hline $\mathrm{n}$ & TOTAL & $04(21,6 \%)$ & $11(52,4 \%)$ & $15(62,5 \%)$ & $30(46,9 \%)$ \\
\hline
\end{tabular}

ESF, escala de severidade da fadiga; FIS, física; MENT, mental. 


\section{DISCUSSÃO}

A fadiga é queixa comum, tanto em indivíduos sadios quanto naqueles portadores de EM. Estudos demonstram que diferentemente do que ocorre em outras situações, na EM ela se mostra mais intensa e frequentemente associada a um grau de incapacidade persistente ${ }^{2,12}$. Embora seja crônica, ela flutua na sua intensidade, sendo mais intensa no final da tarde e com temperaturas elevadas ${ }^{4,5}$. Em aproximadamente 1/3 dos pacientes, a fadiga foi relatada como o primeiro sintoma da doença $a^{4}$, e tem importância fundamental para o estado geral dos pacientes, podendo agravar os demais sintomas da doença $a^{2,5}$.

No grupo estudado, a fadiga foi detectada em $67,4 \%$ dos pacientes, o que está de acordo com os dados de Mainero e col. ${ }^{13}$, que encontraram fadiga em $60,5 \%$ dos pacientes com a forma RR. Outros autores observaram fadiga em número mais elevado de pacientes, porém os estudos incluem pacientes com a forma progressiva da doença ${ }^{4,14}$. Os dados foram semelhantes na comparação entre os grupos com e sem fadiga quanto ao gênero, à média de idade dos pacientes, ou grau de incapacidade funcional, o que está de acordo com os dados de literatura ${ }^{1,4,15}$. No nosso estudo, a duração média da doença, em meses, foi maior no grupo com fadiga $(75,3 \pm 50,2)$ que no grupo sem fadiga $(37,6 \pm$ $37,0)$. Acreditamos que estes dados devam ser melhor analisados, pois esta informação além de nos ser fornecida pelo paciente, é submetida à interpretação do médico, podendo não ser precisa. As controvérsias observadas na literatura, quanto à influência do tempo de aparecimento da doença e a presença de fadiga, devem-se provavelmente à dificuldade em determinar o início da doença, devido as características peculiares da $\mathrm{EM}^{4,12}$.

De acordo com os nossos dados, não há correlação entre a presença de fadiga e de depressão. Embora nossos dados estejam de acordo com os de alguns autores ${ }^{1,15}$, outros mostram haver influência da depressão sobre o aparecimento do sintoma ${ }^{13}$. Nossos achados sugerem que, embora os pacientes com fadiga refiram sintomas semelhantes àqueles observados em pacientes depressivos, apenas uma pequena porcentagem deles apresenta depressão. Na nossa série, a ansiedade está presente em grande número de pacientes com fadiga $(32,8 \%)$ e embora a maioria dos estudos não faça referência a associação entre ansiedade e fadiga, este talvez seja um fator de importância para uma melhor abordagem terapêutica.

Ao analisarmos as características da fadiga de acordo com a sua intensidade, observamos um aumento da média de idade e da duração da doença naqueles casos de fadiga mais intensa. O EDSS também parece estar associado a maior intensidade da fadiga. O EDSS médio dos pacientes com fadiga leve foi $1,8( \pm 1,3)$, enquanto naqueles com fadiga grave foi 3,0 $( \pm 1,4)$. Da mesma forma, enquanto em apenas 5,3\% dos pacientes com fadiga leve o EDSS é maior ou igual a 3,5, no grupo com fadiga grave, $37,5 \%$ apresentam este grau de incapacidade. Estes dados sugerem que a progressão da doença aumenta a severidade da fadiga, o que também foi observado por Cookfair e cols ${ }^{16}$.

A depressão mostrou-se mais frequente naqueles pacientes com fadiga grave, enquanto a ansiedade tem correlação negativa com a maior intensidade do sintoma. A sobreposição de sintomas, pode levar a uma maior dificuldade na identificação da fadiga, aumentando o número de falsos positivos. Este achado, pela relevância que tem na prática clínica, merece estudos mais detalhados.

A EFCm, talvez por ser uma escala geral, não desenvolvida especialmente para os pacientes com EM, mostrou-se menos sensível para detectar fadiga, tendo maior relevância a análise de seus sub-itens, conforme já demonstrado anteriormente ${ }^{5}$. Apenas 30 (46,9\%) dos pacientes também obtiveram escore elevado na EFCm. Maior número de pacientes apresentou escore elevado no subitem fadiga física. A severidade da fadiga parece influenciar o tipo de fadiga apresentada, assim como a sensibilidade da escala. Naqueles pacientes com fadiga leve, a EFCm detectou o sintoma em poucos pacientes. Já nos grupos com fadiga moderada e grave, além de uma maior sensibilidade da escala, observamos uma predominância da fadiga física sobre a mental. 


\section{Conclusão}

Embora em nosso estudo, nos pacientes com EM a fadiga não se correlacione com gênero, idade, depressão ou grau de incapacidade funcional, alguns destes fatores parecem ter relevância quanto à intensidade da fadiga. Esta associa-se a média de idade mais elevada, maior duração da doença e maior grau de incapacidade funcional. Novos estudos estão sendo realizados para que possamos compreender melhor este achado. Aparentemente existem mecanismos centrais e periféricos envolvidos na fisiopatologia da fadiga ${ }^{14,15}$, porém, não é conhecida a relação entre a fadiga observada nos pacientes com EM e a fadiga muscular. O reconhecimento de diversas formas de manifestação da fadiga, com diferentes fisiopatologias, e a influência de fatores psicológicos e imunológicos envolvidos no aparecimento e na intensidade da manifestação da fadiga, nos permitirá atuar de forma mais efetiva no alívio deste sintoma.

\section{REFERÊNCIAS}

1. Krupp LB, Coyle PK. Fatigue in neurologic disease. XLVI American Congress of Neurology, May, 1994.

2. Fisk JD, Ritvo PG, Ross L et al. Measuring the functional impact of fatigue on patients with multiple sclerosis. Can $\mathbf{J}$ Neurol Sci 1994;21:9-14.

3. Iriarte J, Castro P. Propuesta de una nueva escala para evaluacion de la fatiga en pacientes com esclerosis multiple. Neurologia 1994;9:96-100.

4. Krupp LB, Alvarez LA, Larocca NG, et al. Clinical characteristics of fatigue in multiple sclerosis. Arch Neurol 1988;45:435-437.

5. Mendes MF, Tilbery CP, Felipe E. Fadiga e esclerose múltipla: estudo preliminar de 15 casos através de escalas de autoavaliação. Arq Neuropsiquiatr 1996;54 (Suppl 1):96.

6. Poser CM, Paty DW, Scheinberg L, et al. New diagnostic criteria for multiple sclerosis: guidelines for research protocols. Ann Neurol 1983;13:227-231.

7. Krupp LB, LaRocca NG, Muir-Nash J, Steinberg AD. The fatigue severity scale: application to patients with multiple sclerosis and systemic lupus erythematosus. Arch Neurol 1989;46:1121-1123.

8. Chalder T, Berelonitz G, Pawlikowska T, et al. Development of a fatigue scale. J Psychosom Res 1993;37:147-153.

9. Mendes MF, Moreira MA, Tilbery CP, Felipe E - Escalas de auto-avaliação para fadiga: adaptação para a língua portuguesa. Arq Neuropsiquiatr 56(Suppl 1):160.

10. Kurtzke JF. Rating neurologic impairment in multiple sclerosis: an expanded disability status scale. Neurology 1983;33:1444-1452.

11. Botega NJ, Bio MR, Zomignani MA et al - Transtornos do humor em enfermaria de clínica médica e validação de escala de medida de ansiedade e depressão. Rev Saude Publ 1995;29:355-363.

12. Freal JE, Kraft GH, Coryell JK. Symptomatic fatigue in multiple sclerosis. Arch Phys Med Rehabil 1984;65:135-138.

13. Mainero C, Cannoni S, Mancini A, et al. Determinants of fatigue in MS: relationship with clinical subtype and influence on quality of life. Multiple Sclerosis 1999;5(Suppl 1):S6.

14. Iriarte J, Castro P. Correlation between symptom fatigue and muscular fatigue in multiple sclerosis. Eur J Neurol 1998;5:579585.

15. Vercoulen JHMM, Hommes OR, Swanink CMA, et al. The measurement of fatigue in patients with multiple sclerosis. Arch Neurol 1996;53:642-649.

16. Cookfair D, Fischer J, Rudick Ra. Fatigue severity in low disability MS patients participating in a phase III trial of avonex (INF $\beta$-1A) for relapsing multiple sclerosis. Neurology 1997;48(Suppl 2):A173. 